\title{
PREPARO DE PADRÃO SECUNDÁRIO PARA IDENTIFICAÇÃO DE CLORIDRATO DE
}

TRAMADOL

\section{SECONDARY STANDARDT PREPARATION FOR IDENTIFICATION OF TRAMADOL CHLORIDRATE}

\author{
Paula Caroline KLOTH ${ }^{1}$, Aline Tavares HENKE', Maria Madalena GABRIEL ${ }^{2}$, Ricardo \\ WAGNER ${ }^{2}$
}

1 - Acadêmicas do Curso de Farmácia, Universidade Federal do Paraná, Curitiba, Paraná, Brasil

2 - Professores de Toxicologia, Curso de Farmácia, Universidade Federal do Paraná, Curitiba, Paraná, Brasil

Autor para correspondência: ricardo.wagner@ufpr.br

\section{RESUMO:}

O medicamento cloridrato de tramadol, apresenta diversos problemas sociais devido a sua possível toxicidade e potencial de abuso quando utilizado por períodos longos. Desta maneira, para a detecção dessa substância em líquidos biológicos os laboratórios necessitam utilizar padrões de identificação. Muitas vezes esses padrões apresentam um custo elevado e são de difícil obtenção. Em vista a essas dificuldades, o presente trabalho teve como objetivo o desenvolvimento de um padrão secundário a partir de um medicamento doado ao laboratório de Toxicologia da UFPR. Para o desenvolvimento, a substância cloridrato de tramadol foi extraída a partir de 8 comprimidos de Tramal Retard® de $100 \mathrm{mg}$. Como resultado da extração, obteve-se um rendimento de 89,37\%. A substância extraída apresentou um pico de absorção característico no espectro de absorção ultravioleta em $275 \mathrm{~nm}$. A análise por CLAE-FLD mostrou apenas um pico único nas condições analíticas utilizadas. O teor determinado por titulação com ácido perclórico foi de $94,39 \%$. O padrão secundário obtido foi aplicado em uma análise qualitativa por cromatografia em camada delgada frente a uma urina sabidamente positiva para uso de tramadol e apresentou Rf de 0,77, comparável ao tramadol encontrado na urina. Por fim, conclui-se que o padrão desenvolvido está apto para ser utilizado na rotina para métodos de triagem na identificação do cloridrato de tramadol em aulas práticas de Toxicologia.

Palavras chave: padrão secundário, cloridrato de tramadol.

ABSTRACT: The drug tramadol hydrochloride, presents several social problems due to its possible toxicity and potential abuse when used for long periods. In this way, for the detection of this substance in biological fluids the laboratories need to use standards to identificate the substance. Often these standards are costly and difficult to obtain. In view of these difficulties, the present work had the objective of developing a secondary standard from a drug donated to the UFPR toxicology laboratory. For the development, tramadol hydrochloride was extracted from 8 Tramal Retard $\Theta 100 \mathrm{mg}$ tablets. As a result of the extraction, a yield of $89.37 \%$ was obtained. The extracted substance showed a characteristic absorption peak in the ultraviolet absorption spectrum at $275 \mathrm{~nm}$. Analysis by HPLC-FLD showed only a single peak under the analytical conditions used. The content determined by titration with perchloric acid was $94.39 \%$. The secondary standard obtained was applied in 
a qualitative analysis by thin layer chromatography against urine known to be positive for tramadol and presented $\mathrm{Rf}$ of 0,77 comparable to tramadol found in urine. Finally, it is concluded that the standard developed is apt to be used routinely for screening methods in the identification of tramadol hydrochloride in practical Toxicology classes.

Key words: secondary standart, tramadol hydrochloride.

\section{INTRODUÇÃO}

Em razão do seu potencial de abuso e intoxicação pelo medicamento cloridrato de tramadol é passível de investigação toxicológica para verificar uma possível intoxicação por esta substância. Para a detecção dessa substância em líquidos biológicos os laboratórios necessitam utilizar padrões de identificação e, desta forma, existem dois tipos de padrões que podem ser utilizados: o padrão primário e o padrão secundário. O padrão primário é uma substância cujo elevado grau de pureza e autenticidade foram demonstrados por meio de testes analíticos. O padrão secundário é uma substância de qualidade e pureza estabelecidas, após comparação com um padrão de referência primário. (ANVISA, 2010).

O padrão primário é um reagente puro para ser pesado e utilizado de forma direta. Sabe-se que a precisão do método analítico que irá se realizar encontra-se relacionada com as propriedades deste composto. O padrão primário deve apresentar alta pureza (99,9\% ou superior), de fácil obtenção, solubilidade, dessecação e conservação, apresentar estabilidade à atmosfera, não ser higroscópico e possui grande massa molar para minimizar erros relativos à pesagem padrão. (SKOOG et al., 2004).

Poucos compostos preenchem esses critérios, e somente um número limitado de padrão primário está disponível comercialmente. Como consequencia, os compostos menos puros como os padrões secundários são utilizados no lugar de um padrão primário. (SKOOG et al., 2004). No que diz respeito ao padrão secundário, sabe-se que são substâncias que possuem concentração determinada através de análise química. A pureza dessa substância deverá ser estabelecida por análise cuidadosa. (SKOOG et al., 2004).

$\mathrm{O}$ cloridrato de Tramadol possui a fórmula química $\mathrm{C}_{16} \mathrm{H}_{25} \mathrm{NO}_{2} . \mathrm{HCl}$. Sua massa molar é de $299,84 \mathrm{~g} / \mathrm{mol}$. O ponto de fusão dessa substância é de $180{ }^{\circ} \mathrm{C}$ a $184{ }^{\circ} \mathrm{C}$ e ponto de ebulição $388,1 \pm 22^{\circ} \mathrm{C}$ em $760 \mathrm{mmHg}$. É solúvel em água, etanol e metanol e muito pouco solúvel em acetona. (ANVISA, 2010).

De acordo com a Farmacopeia Brasileira 5a edição (2010, p. 870) o cloridrato de tramadol contém, no mínimo, 99,0\% e, no máximo, 101,0\% de $\mathrm{C}_{16} \mathrm{H}_{25} \mathrm{NO}_{2} . \mathrm{HCl}$ em relação a substância anidra, quando se trata de SQR (substância química de referência). tramadol possui pKa de 9,41 e o seu coeficiente de partição água/n-octanol é 1,35 em pH 
7,0. (SHINDE; GARALA; MORE, 2008; HABIBOLLAHI et al., 2015). Tendo visto o que foi exposto o objetivo deste trabalho foi obter um padrão secundário de cloridrato de tramadol a partir de comprimidos e determinar o grau de pureza através de métodos químicos e espectroscópicos.

\section{MATERIAL E MÉTODOS}

O material utilizado para a produção de padrão secundário de Cloridrato de Tramadol foram 8 comprimidos de $100 \mathrm{mg}$ do medicamento Tramal retard® doado para 0 laboratório de Toxicologia Clínica, curso de Farmácia da Universidade Federal do Paraná.

\subsection{Extração a partir do comprimido}

A extração foi feita pulverizando os 8 comprimidos em gral e pistilo até a obtenção de um pó fino. Transferiu-se então esse material para um tubo Falcon de $50 \mathrm{~mL}$ e em seguida foram adicionados $30 \mathrm{~mL}$ de metanol, solvente este escolhido de acordo com a solubilidade do composto. A solução foi agitada por 5 minutos em vórtex. Posteriormente, o material foi centrifugado por 5 minutos à 2500 rpm. Ao precipitado foram adicionados mais $30 \mathrm{~mL}$ de metanol e feita uma segunda extração. A fase orgânica foi filtrada em papel filtro e transferida para um béquer de $100 \mathrm{~mL}$. Este foi levado em banho maria até a completa evaporação do solvente. Após evaporação do solvente o material foi ressuspenso em água deionizada, congelado e liofilizado. Após liofilização o material foi pesado e calculado o rendimento da extração.

\subsection{Análise do ponto de fusão}

Para o ponto de fusão pegou-se uma pequena quantidade do material liofilizado extraído transferindo-o para 2 capilares. Esses capilares foram colocados no equipamento METTLER TOLEDO modelo MP70 Melting Point System, configurando a faixa de fusão de $170{ }^{\circ} \mathrm{C}$ a $185^{\circ} \mathrm{C}$ variando $1^{\circ} \mathrm{C}$ por minuto.

\subsection{Análise por cromatografia em camada delgada}

Para esta análise preparou-se $10 \mathrm{~mL}$ uma solução $1 \mathrm{mg} \mathrm{mL}^{-1}$ do tramadol liofilizado 
em metanol. Para a cromatografia em camada delgada utilizou-se duas cromatoplacas de sílica gel 60 GF254, 250 $\mu$ m de espessura em placa de alumínio marca MN e como fase móvel metanol:hidróxido de amônio $(99: 1, v / v)$. O tempo de saturação da cuba foi de 30 minutos e o número de aplicações da solução padrão foram de 15, 30 e 40 aplicações, utilizando-se de um microcapilar. A corrida cromatográfica foi de $9 \mathrm{~cm}$. Uma das cromatoplacas foi revelada com iodo platinado e a outra com reagente de Dragendorff. (MEYYANATHAN; KUMAR; SURESH, 2003; VENKATESHWARLU et al., 2008).

\subsection{Análise por cromatografia líquida de alta eficiência acoplada a detector de fuorescência (clae-fld)}

Para o preparo da amostra pesou-se $10 \mathrm{mg}$ do material liofilizado e em seguida foi feita uma diluição em metanol grau HPLC até obter uma concentração de 0,003 mg/mL. Para a análise em CLAE utilizou-se o cromatógrafo Varian ProStar, uma coluna C18 Pursuit XRS Ultra $(2,8 \mu \mathrm{m}, 150 \times 20 \mathrm{~mm})$; fluxo da fase móvel de $0,3 \mathrm{~mL} \mathrm{~min}^{-1}$. Utilizou-se um sistema isocrático com fase móvel de ácido tricloroacético a 0,2\% ( $p / v)$ e acetonitrila (705:295). A análise foi feita em duplicata, injetando $20 \mu \mathrm{L}$ da amostra de concentração $0,010 \mathrm{mg} \mathrm{mL}^{-1}$ e $20 \mu \mathrm{L}$ da amostra de concentração $0,003 \mathrm{mg} \mathrm{mL}^{-1}$. Os detectores utilizados para análise foram ultravioleta em $270 \mathrm{~nm}$ e FLD 275/300 nm. (ANVISA, 2010).

\subsection{Doseamento do tramadol obtido da extração}

O doseamento foi realizado através de uma titulação ácido-base em meio não aquoso, utilizando ácido perclórico 0,1 $\mathrm{M}$ como titulante, conforme preconizado pela Farmacopeia Brasileira, 5a edição. Para a padronização do ácido perclórico, pesou-se exatamente cerca de $204 \mathrm{mg}$ de biftalato de potássio dissolvendo-o em $80 \mathrm{~mL}$ de anidrido acético em um erlenmeyer de $250 \mathrm{~mL}$. Completou-se então uma bureta de $50 \mathrm{~mL}$ com ácido perclórico 0,1 M. Como solução indicadora utilizou-se cristal violeta. Após a padronização, calculou-se o fator de correção do ácido perclórico, obtendo-se assim uma solução $0,1 \mathrm{M}$ SV (solução volumétrica). Para a titulação do cloridrato de tramadol pesou-se exatamente cerca de $90 \mathrm{mg}$ da amostra liofilizada de cloridrato de tramadol dissolvendo-o em $80 \mathrm{~mL}$ de anidrido acético em um erlenmeyer de $250 \mathrm{~mL}$. Completou-se então uma bureta de $50 \mathrm{~mL}$ com ácido perclórico 0,1 M SV. Como solução indicadora utilizou-se cristal violeta. Após a titulação, calculou-se a dose de cloridrato de tramadol presente na amostra liofilizada. (ANVISA, 2010). 


\subsection{Utilização do padrão secundário de tramadol obtido}

Para verificar a aplicabilidade do padrão de tramadol obtido foi realizada uma pesquisa toxicológica utilizando uma urina de um indivíduo que fez uso de tramadol durante uma semana consecutiva, ou seja, uma urina comprovadamente positiva para tramadol. Este material foi cedido para a disciplina de Toxicologia e pode ser utilizado neste experimento.

Para a extração foi utilizado um erlenmeyer de $125 \mathrm{~mL}$, adicionou-se $10 \mathrm{~mL}$ de urina com o auxílio de uma pipeta. Adicionou-se então gotas de hidróxido de sódio 10\% até obter $\mathrm{pH}$ entre 9-10, medido com fita indicadora de $\mathrm{pH}$. Em seguida, adicionou-se $15 \mathrm{~mL}$ de clorofórmio no erlenmeyer, agitando-o por 5 minutos. Após um curto repouso, retirou-se a camada clorofórmica para um bécker de $50 \mathrm{~mL}$. Repetiu-se a extração com mais $15 \mathrm{~mL}$ de clorofórmio, transferindo-se novamente a camada clorofórmica para o mesmo béquer. Filtrou-se então a camada orgânica coletada por sulfato de sódio anidro. O béquer foi levado em seguida ao banho-maria até evaporação do solvente. Ressuspendeu-se o resíduo com 0,5mL de clorofórmio. (MOFFAT, 1986).

Para a CCD utilizou-se duas cromatoplacas de Sílica gel 60 GF254 $250 \mu \mathrm{m}$ de espessura em base de alumínio. Nesta placa foram feitos 2 pontos equidistantes, em um deles, aplicou-se o resíduo extraído de urina, 40 aplicações, e o outro ponto o padrão secundário de tramadol 1mg/mL, 40 aplicações, com microcapilar. Utilizou-se fase móvel de metanol:hidróxido de amônio $(99: 1, v / v)$. O tempo de saturação foi de 30 minutos. Uma das cromatoplacas foi revelada com iodo platinado e a outra com reagente de Dragendorff. (MOFFAT, 1986)

\section{RESULTADOS E DISCUSSÃO}

A quantidade extraída dos 8 comprimidos de $100 \mathrm{mg}$ foi $715 \mathrm{mg}$. O rendimento teórico deveria ser de $800 \mathrm{mg}$, com isso o rendimento prático calculado foi de $89,37 \%$, esta perda possivelmente ocorreu por solubilização incompleta do material a ser extraído ou por perdas durante a manipulação do material. Após este cálculo foi realizado o ponto de fusão e quando a temperatura alcançou $182,2^{\circ} \mathrm{C}$ a amostra estava completamente fundida. De acordo com a Farmacopéia Brasileira $5^{\mathrm{a}}$ ed. (2010) o ponto de fusão do cloridrato de tramadol é de $180{ }^{\circ} \mathrm{C}$ a $184^{\circ} \mathrm{C}$ em $760 \mathrm{mmHg}$. Desta forma, o ponto de fusão da amostra liofilizada coincide com valores encontrados na literatura, indicando grau de pureza do padrão 
secundário de tramadol obtido.

\subsection{Cromatografia em camada delgada}

Para a avaliação de padrão secundário obtido foi realizada uma cromatografia em camada delgada com dois sistemas de revelação diferentes. Após a revelação das duas cromatoplacas, determinou-se que ambas as revelações foram satisfatórias e foi possível observar apenas uma mancha referente ao padrão de tramadol.

Quanto ao número de aplicações, verificou-se que 15 aplicações não foram suficientes para a revelação com iodo platinado (FIGURA 1A), diferente da revelação com Dragendorff (FIGURA 1B), mostrando desta forma que o reativo de Dragendorff apresenta uma sensibilidade maior para a revelação de pequenas quantidades de tramadol. Definiuse então que 30 ou 40 aplicações seriam o ideal para as análises posteriores onde se usará este padrão secundário na concentração de $1 \mathrm{mg} \mathrm{mL}^{-1}$. Quanto ao Rf encontrado, foi de 0,77 para todas as aplicações nas duas placas. O padrão de revelação pode ser visualizado na (FIGURA 1).

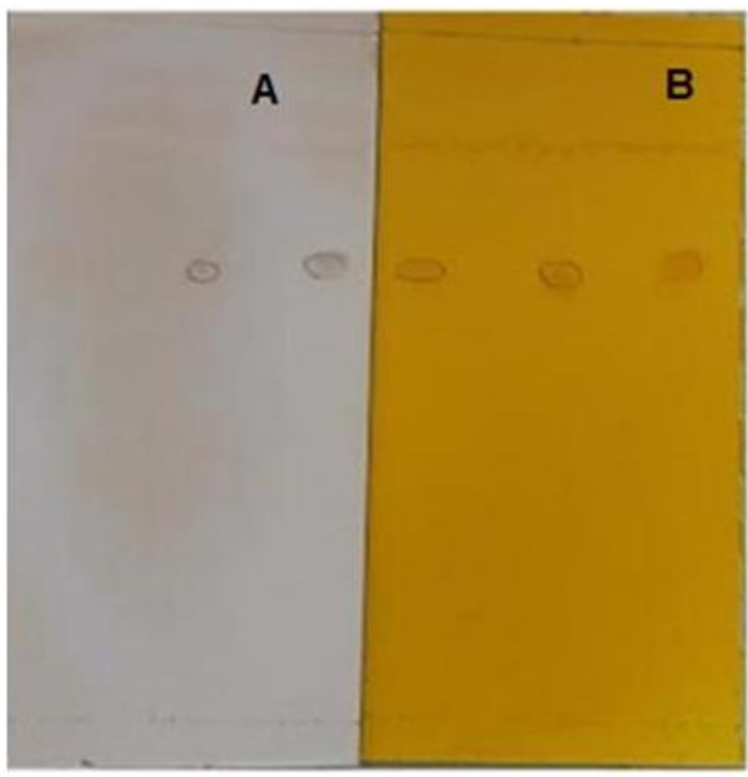

FIGURA 1 - CROMATOGRAFIA EM CAMADA DELGADA DO EXTRATO LIOFILIZADO DE CLORIDRATO DE TRAMADOL. (A) REVELAÇÃO COM IODO PLATINADO, (B) REVELAÇÃO COM DRAGENDORFF. 


\subsection{Cromatografia líquida de alta eficiência}

A análise em CLAE-FLD foi realizada para analisar o comportamento cromatográfico da amostra nas condições utilizadas e para poder verificar se o grau de pureza estava de acordo com o doseamento, que será discutido no próximo tópico. Para isso, injetou-se $20 \mu \mathrm{L}$ da amostra do padrão secundário de tramadol na concentração 0,010 $\mathrm{mg} \mathrm{mL}^{-1}$. Após uma corrida de 10 minutos, obteve-se um único pico estreito conforme a (FIGURA 2), com tempo de retenção de 4,4 minutos.

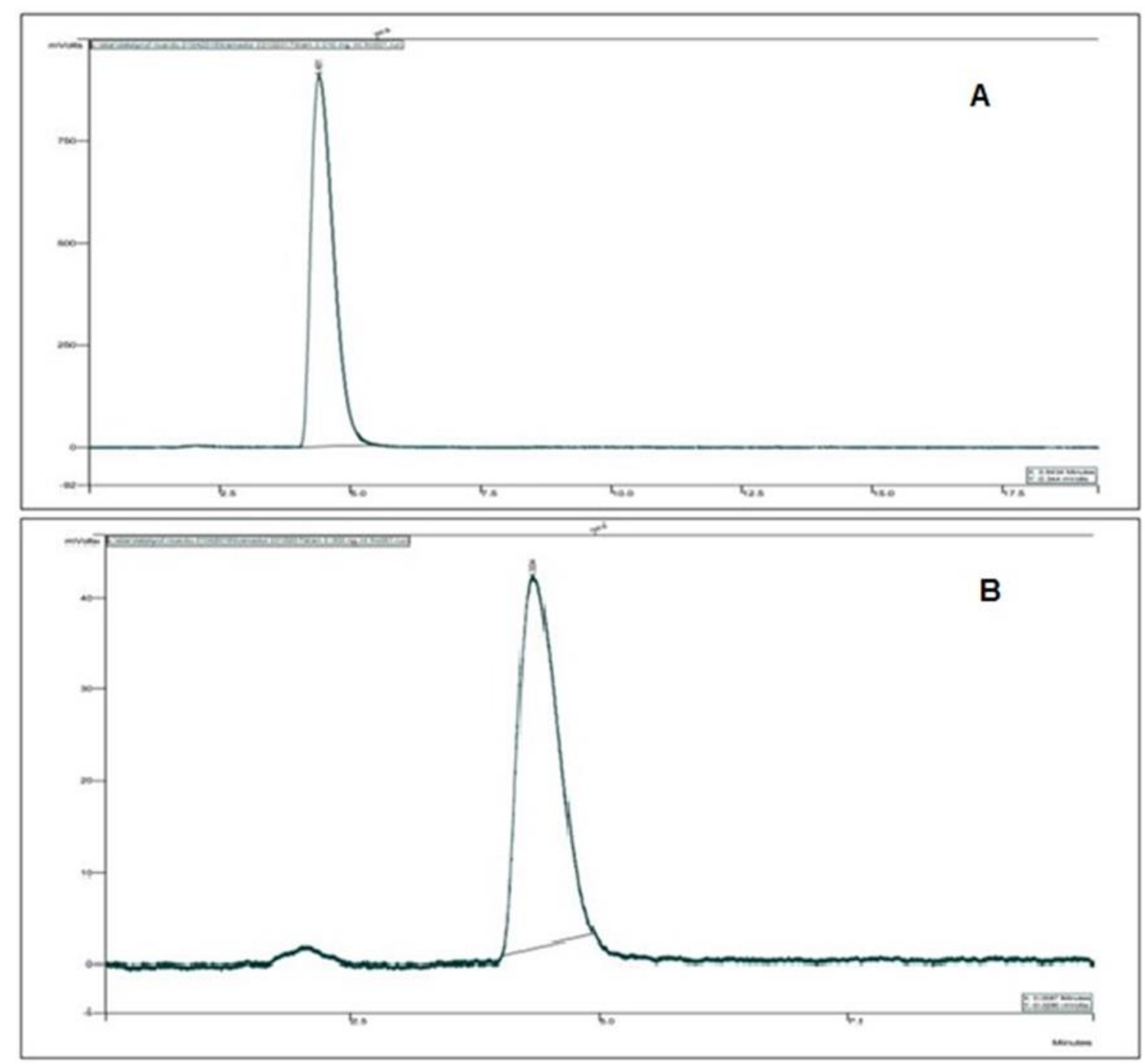

FIGURA 2 - CROMATOGRAFIA LÍQUIDA DE ALTA EFICIÊNCIA PADRÃO SECUNDÁRIO DE CLORIDRATO DE TRAMADOL (A) EM CONCENTRAÇÃO 0,010 mg mL-1 E (B) EM CONCENTRAÇÃO $0,003 \mathrm{mg} \mathrm{mL-1.}$ 
Em seguida, sobre os mesmos parâmetros cromatográficos, injetou-se $20 \mu \mathrm{L}$ da amostra de concentração $0,003 \mathrm{mg} \mathrm{mL}^{-1}$, obtendo-se um pico único e estreito. Nas duas análises foi possível observar picos únicos com boa resolução cromatográfica e iguais tempos de retenção.

Após a análise da amostra em CLAE, obtivemos um único pico característico do cloridrato de tramadol, indicando a pureza qualitativa da amostra, pois mesmo em uma concentração mais alta não foi observado nenhum outro pico interferente. A amostra de concentração $0,010 \mathrm{mg} \mathrm{mL}^{-1}$ mostrou um tempo de retenção de 4,401 minutos, enquanto a amostra de concentração $0,003 \mathrm{mg} \mathrm{mL}^{-1}$ mostrou um tempo de retenção de 4,334 minutos, esta pequena diferença pode ser relativa ao modo de injeção que foi manual e não automatizado.

\subsection{Doseamento do padrão secundário}

Antes do doseamento foi realizada a padronização do ácido perclórico $0,1 \mathrm{M}$ com biftalato de potássio. Para isso, fez-se uma titulação em triplicata, pesando-se com exatidão 204,1 mg, 204,1 mg e 203,9 mg de biftalato de potássio previamente dessecado em estufa por $1 \mathrm{~h}$ a $110^{\circ} \mathrm{C}$ seguindo as informações da Farmacopeia $5^{\circ}$ ed. (2010). Calculou-se então o volume teórico para cada uma das massas pesadas, sabendo-se que a reação estequiométrica é 1:1 entre o ácido perclórico e o biftalato de potássio. Em seguida, foi feita a titulação de cada uma das massas pesadas, obtendo-se 9,6, 9,7 e 9,3 mL de volume prático, respectivamente, para cada amostra. Calculou-se então 3 fatores de correção, dividindo-se o valor do volume teórico pelo valor do volume prático, obtendo-se ao final uma média dos 3 valores de 1,0482 (TABELA 1).

TABELA 1 - DADOS DA PADRONIZAÇÃO DO ÁCIDO PERCLÓRICO 0,1M COM BIFTALATO DE POTÁSSIO.

\begin{tabular}{cccc}
\hline Massa $\mathrm{C}_{8} \mathrm{H}_{5} \mathrm{KO}_{4}(\mathrm{~g})$ & $\begin{array}{c}\text { Volume teórico de } \\
\mathrm{HClO}_{4} 0,1 \mathrm{M}(\mathrm{mL})\end{array}$ & $\begin{array}{c}\text { Volume Prático de } \\
\mathrm{HClO}_{4} 0,1 \mathrm{M}(\mathrm{mL})\end{array}$ & $\begin{array}{c}\text { Fator de correção (Fc) } \\
\text { obtido }\end{array}$ \\
\hline 0,2041 & 9,9941 & 9,6 & 1,0410 \\
0,2041 & 9,9941 & 9,7 & 1,0303 \\
0,2039 & 9,9843 & 9,3 & 1,0735 \\
\hline
\end{tabular}


Para o doseamento, a titulação foi realizada em triplicata, pesando exatamente 90,4 mg, 92,3 mg e 92,4 mg da amostra liofilizada de cloridrato de tramadol. Em seguida, foi feita a titulação de cada uma das massas pesadas, obtendo-se 3,2; 3,1 e 3,1 mL de volume prático respectivamente para cada amostra conforme a tabela abaixo (TABELA 5). Calculou-se então o teor de cada amostra doseada, obtendo-se uma média dos 3 valores de 94,3994\% conforme (TABELA 2). Após o doseamento verificou-se que a amostra de padrão secundário obtida possui uma boa pureza para ser utilizada como padrão secundário.

TABELA 2 - DADOS OBTIDOS DO DOSEAMENTO DO CLORIDRATO DE TRAMADOL

\begin{tabular}{lccc}
\hline $\begin{array}{l}\text { Massa de tramadol } \\
\text { pesada }(\mathrm{g})\end{array}$ & $\begin{array}{c}\text { Volume prático de } \mathrm{HClO}_{4} \\
0,1 \mathrm{M} \mathrm{SV}(\mathrm{mL})\end{array}$ & $\begin{array}{c}\text { Volume prático x } \\
\text { fator de correção }\end{array}$ & Teor (\%) \\
\hline 0,0904 & 3,2 & 3,35456 & 97,7349 \\
0,0923 & 3,1 & 3,24973 & 92,7317 \\
0,0924 & 3,1 & 3,24973 & 92,7317 \\
\hline & & Média do teor & $\mathbf{9 4 , 3 9 9 4}$ \\
\hline
\end{tabular}

\subsection{Utilização do padrão secundário de tramadol em análise toxicológica a partir de matriz biológica urina por ccd}

A fim de verificar se o padrão secundário produzido é capaz de ser utilizado em rotina de laboratórios, foi realizada uma extração líquido-líquido do cloridrato de tramadol em uma amostra de urina positiva para esta substância e comparou-se o resultado com o padrão secundário de tramadol obtido neste trabalho, por cromatografia em camada delgada. A matriz biológica utilizada foi a urina em virtude de que é uma das amostras mais comuns utilizadas para o monitoramento do uso do medicamento, e também para verificar possível intoxicação, por ser de fácil acesso e com menor número de interferentes.

Após a extração do cloridrato de tramadol da urina foram preparadas duas 2 cromatoplacas, fazendo-se 40 aplicações da amostra e 30 aplicações do padrão em cada cromatoplaca. Após a corrida comparou-se então os pontos conforme a (FIGURA 3). O ponto $\mathrm{P}$ corresponde à aplicação o padrão secundário de tramadol. O ponto A corresponde à aplicação da amostra. 
Podemos observar que tanto em uma placa quanto na outra, é possível observar o aparecimento da mancha referente ao padrão $(P)$ com $R f$ de 0,77 . A amostra $(A)$ também apresenta a presença uma mancha sugestiva de tramadol com $\mathrm{Rf}$ de 0,77 . Outra mancha pode ser observada na amostra com $\mathrm{Rf}$ de 0,5 . Esta mancha pode ser entendida como um produto de biotransformação do tramadol, uma vez que em sua toxicocinética é relatada a excreção de $60 \%$ sob a forma de metabólitos livres e conjugados. Desta forma na urina podemos encontrar tanto o tramadol inalterado e quanto seus produtos de biotransformação. (MOFFAT, 1986).

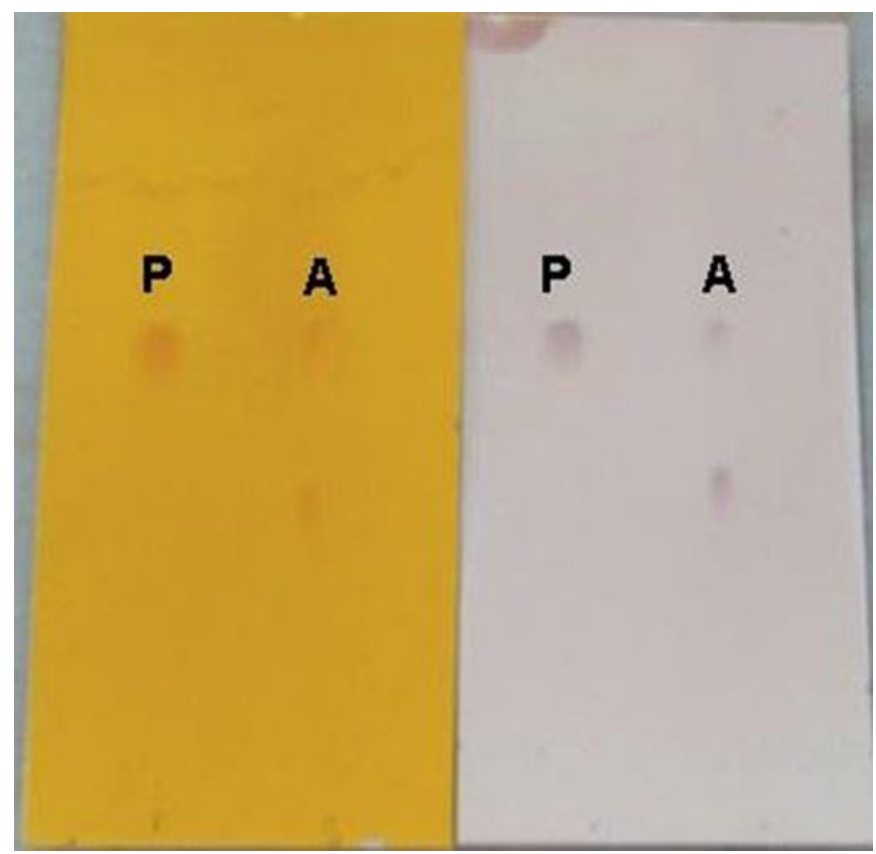

FIGURA 3 - CROMATOGRAFIA EM CAMADA DELGADA DA AMOSTRA BIOLÓGICA DE USUÁRIO DE CLORIDRATO DE TRAMADOL (A) EM COMPARAÇÃO COM O PADRÃO SECUNDÁRIO DO CLORIDRATO DE TRAMADOL $(\mathrm{P})$.

\section{CONSIDERAÇÕES FINAIS}

Após todas as análises realizadas com o padrão secundário de tramadol obtido da extração do medicamento Tramal Retard® 100 mg, concluímos que o mesmo encontra-se apto para a sua utilização como padrão secundário. Isto por que, de acordo com os resultados apresentados neste trabalho, o padrão secundário obtido apresentou um pico de absorção característico em espectro de absorção ultravioleta, um pico único na análise em CLAE-FLD, e grau de pureza razoável, com o doseamento que mostrou um teor de 
$94,39 \%$. Outro ponto que torna esta substância purificada apta para a sua utilização como padrão secundário, é a sua reprodutibilidade quando utilizado em CCD, mostrando que o mesmo poderia ser utilizado na rotina de um laboratório para triagem para presença de cloridrato de tramadol utilizando-se cromatografia em camada delgada ou até mesmo HPLC para estas análises.

\section{REFERÊNCIAS}

ANVISA. AGÊNCIA NACIONAL DE VIGILÂNCIA SANITÁRIA. Farmacopeia Brasileira, volume 2. 5를. Brasilia, 2010. p. 870-871.

HABIBOLLAHI, S.; TAVAKKOLI, N.; NASIRIAN, V.; KHANI, H. Determination of Tramadol by Dispersive Liquid-Liquid Microextraction Combined with GC-MS. Journal of Chromatographic Science. v. 53, p. 655-661, 2015.

JORK, H. et al. Thin-Layer Chromatography - Reagents and Detection Methods. Weinheim: Vch Verlagsgesellschaft Mbh, v. 1a, 1990.

MEYYANATHAN, S. N.; KUMAR, P.; SURESH, B. Analysis of tramadol in pharmaceutical preparations by high performance thin layer chromatography. J. Sep. Sci. v. 26, p. 13591362, 2003.

SHINDE, A. J.; GARALA, K. C.; MORE, H. N. Development and characterization of transdermal therapeutics system of tramadol hydrochloride. Asian Journal of Pharmaceutics. October-December, p. 265-269, 2008.

SKOOG. D. A. et al. Métodos titulométricos; Titulometria de precipitação. In: Fundamentos de química analítica. 8. ed. São Paulo: Bookman, 2005. p. 328.

VENKATESHWARLU, K.; REDDY Y.N.; SRISAILAM, K.; RAJKUMAR, V.; PAI, M.G. Determination of tramadol in capsules by high performance thin layer chromatography densitometry. Current Trends in Biotechnology and Pharmacy, v.. 2 (3), p. 421-425, 2008.

WALL, P. E. Thin-layer Chromatography. Cambridge: The Royal Society of Chemistry, 2005. 